\title{
Comparison of Recovery and Postoperative Analgesia between Thoracic Epidural Anaesthesia (TEA) plus General Anaesthesia and General Anaesthesia alone, in Patients Undergoing Off-Pump Coronary Artery Bypass (OPCAB) Surgery
}

\author{
S Ferdous, S Hossain, N Haque, AYFE Chowdhury \\ Department of Anaesthesia, NICVD, Dhaka.
}

\begin{abstract}
:
Keywords:

Background: In recent years, high thoracic anaesthesia (HTEA) combined with general anaesthesia Thoracic epidural anaesthesia, $O P C A B$, Postoperative analgesia. has been extensively studied in patients undergoing coronary artery bypass graft surgery with or without cardiopulmonary bypass. Off-pump coronary artery bypass grafting was implemented to reduce trauma of surgical coronary revascularization by avoiding extracorporeal circulation. TEA in combination with GA further reduces intraoperative stress resulting in more rapid extubation and significantly better pain relief in patients undergoing off-pump coronary artery bypass grafting $(O P C A B)$. In addition, common postoperative complications are also decreased.
\end{abstract}

Methodology: 40 male patients aged between 40-65 years with CAD undergoing OPCAB surgery at NICVD from April 2006 to October 2008 were randomly divided in two groups. Group A received epidural analgesic drugs through an indwelling catheter introduced before induction of anesthesia while group B patients received standard general anesthesia (GA) alone. Group A patients received continuous epidural analgesia delivered through the indwelling catheter with a mixture of Lignocaine (2\%) $20 \mathrm{ml}+$ Bupivacaine (0.5\%) $20 \mathrm{ml}+$ Fentanyl $50 \mathrm{gm} 1 \mathrm{ml}+$ normal saline q.v. $50 \mathrm{ml}$ at the rate of 1-2 ml/hour by a syringe pump for up to 72 hours. Group B patients received conventional intermittent narcotics (Morphine)/NSAIDS (Ketorolac/Diclofen) for up to 72 hours.

Results: Haemodynamic parameters of all patients in both the groups were within acceptable range throughout operations. Incidence of cardiac dysrhythmias was less in patients of group A. Group A patients showed faster recovery and better analgesia compared to Group B patients. There was also decreased incidence of nausea, vomiting, arrhythmia, $\mathrm{O}_{2}$ desaturation, confusional states, renal failure and requirement of iontropic support in ICU in Group A patients. TEA for postoperative analgesia was safe and well accepted by the patients. There was no complication related to epidural anesthesia in any patient.

Conclusion: TEA in general anesthesia provides faster recovery and effective analgesia. Nonetheless, the actual and potential risks of TEA during cardiac surgery should not be underestimated.

(Cardiovasc. j. 2010; 2(2) : 156-162)

\section{Introduction:}

CABG is the most frequently performed cardiac surgical procedure worldwide. ${ }^{1}$ In the last decades, there has been renewed interest in performing CABG without cardiopulmonary bypass $(\mathrm{CPB})^{2}$ because of advent of new myocardial stabilization devices. ${ }^{3}$ Avoiding CPB eliminates aortic cannulation and cross clamping and is expected to reduce systemic inflammatory response, coagulation disorders and multiple organ dysfunction, ${ }^{4}$ postoperative atrial fibrillation, stroke, ${ }^{5}$ shorter hospital stay, early hospital discharge and outcome. With the advent of new instruments, multivessel bypass is now performed on the beating heart. OPCAB surgery may be associated with decreased postoperative morbidity and reduced total costs. ${ }^{6}$ Its appears to produce better results than conventional surgery in high

Address of Correspondence: Dr. Shahnaz Ferdous, Department of Anaesthesiology, National Institute Cardiovascular Diseases, Dhaka, Bangladesh. 
risk patient populations, especially for patients with compromised cardiac function or coagulation disorders and severe co morbidities including old age.

TEA in combination with general anesthesia is a recently introduced technique for OPCAB surgery. It faces growing interest as an adjuvant anesthetic and postoperative analgesic regimen. ${ }^{7}$ It would be one of the most effective methods of anesthesia for OPCAB surgery as it combines the advantages of the two techniques while minimizing their drawbacks.

TEA in combination with general anesthesia has been shown to provide intense perioperative analgesia, reliable attenuation of the stress response to $\mathrm{CPB}$, thoracic cardiac sympatholysis and decreased coronary vascular resistance; decreased heart rate and use of beta-blockers after CPB; increased myocardial oxygen supply. ${ }^{8}$ TEA provides superlative analgesia, obviating the need for opioids and reducing the duration of mechanical ventilation, facilitating early tracheal extubation compared to standard general anesthesia alone..$^{9,10}$ In addition to these intraoperative advantages, postoperative pain management is facilitated by continuous epidural administration of analgesics, which improves postoperative mobilization and recovery. ${ }^{11}$

In the present study, TEA in combination with general anesthesia was used in OPCAB surgery. Haemodynamic stability was studied in both intraoperative and postoperative period to find out an effective method of anesthesia for OPCAB surgery.

We hypothesize that TEA may cause faster recovery and better postoperative analgesia. Thus, the aim of this study was to investigate the effect of TEA on recovery and postoperative analgesia in patients with $\mathrm{CAD}$ undergoing elective OPCAB surgery.

\section{Materials and Methods:}

Between April 2006 and October 2008, 40 male patients aged between 40-65 years, were randomly assigned into two groups for elective OPCAB surgery. Written informed consent was obtained from all patients. The local ethics committee approved the study.
Patients were premedicated by oral Midazolam (7.5 $\mathrm{mg}$ at H/S) and Inj. Morphine $7.5 \mathrm{mg} \mathrm{i} / \mathrm{m}$ one hour before operation.

Patient's selection criteria included age $>18$ yrs, sinus rhythm on ECG preoperatively, restriction to significant $(>70 \%)$ stenosis of the left anterior coronary artery (LAD), diagonal branches or the right coronary artery as well as a cooperative patient. Presence of co morbidity did not affect the patient selection except for impaired lung function $\left(\mathrm{FEV}_{1}<50 \%\right)$. The exclusion criteria from the study were on oral anticoagulation and coagulopathy. Elective OPCAB were performed through complete median sternotomy.

Group A: High TEA + GA ( $n=15)$

Position: left lateral

Space: T1-T2 interspace with 18 G Touhy needle \& catheter.

Direction of the catheter: Cranial

Length of catheter into epidural space: 3-4 cm.

Drugs: Inj. Bupivacaine (0.5\% plain) $8-10 \mathrm{ml}$ +10 ìg Fentanyl

Top-up bolus doses of $4 \mathrm{ml}$ of Bupivacaine, 0.5\% were administered hourly during the operation. The TE infusion was continued with a mixture of lignocaine (2\%) $20 \mathrm{ml}+$ Bupivacaine (0.5\%) $20 \mathrm{ml}+$ fentanyl 50 ìg $1 \mathrm{ml}+$ normal saline q.v. $50 \mathrm{ml}$ at the rate of $1-2 \mathrm{ml} / \mathrm{hour}$ by a syringe pump and also for postoperative pain management up to 72 hours. The catheter was removed on postoperative day 4.

GA: standard GA, Maintained with halothane, IV Fentanyl, and Pancuronium.

Group B: GA alone: same as group A ( $\mathrm{n}=15)$.

Maintained with halothane + IV Midazolam, Morphine, Fentanyl, Pancuronium.

Postoperative analgesia was provided by conventional intermittent narcotics (Morphine) / NSAIDS (Ketorolac, Diclofenac) for up to 72 hours.

High TEA was used in group A only in combination with GA. In the operating room one hour before cardiac surgery, the patient was placed in left lateral position. Epidural space was identified using $18 \mathrm{G}$ (B Braun) Touhy needle at the interspace between T1-2 space employing the median 
approach and the loss of resistance technique using air under local anesthesia. The catheter was directed cranially and advanced $3-4 \mathrm{~cm}$ within the epidural space. After confirmation of the correct position of the catheter, $2 \%$ lignocaine $2.5 \mathrm{ml}$ was administered as a test dose. After positioning of the patient in supine position and 3-5 minutes of test dose Inj. Bupivacaine 0.5\%-5 ml and Fentanyl $2 \mu \mathrm{g} / \mathrm{ml}$ was injected via the epidural catheter.

Group B patients were received conventional intravenous general anesthesia. After preoxygenation, induction of general anesthesia was done with diazepam/ Midazolam $0.01 \mathrm{mg} / \mathrm{kg}$, morphine $0.2-0.3 \mathrm{mg} / \mathrm{kg}$ and Pancuronium $0.1 \mathrm{mg} /$ $\mathrm{kg}$, Thiopentone sodium $2-3 \mathrm{mg} / \mathrm{kg}$ was added before laryngoscopy and intubation. A Glyceryl Trinitrate infusion (at a rate of $0.1-10 \mu \mathrm{g} / \mathrm{kg} / \mathrm{min}$ ) was started from the syringe pump beforehand to prevent coronary spasm and increase in heart rate. Anesthesia was maintained with halothane $0.5 \%$ $1 \%$ in oxygen and nitrous oxide $70 \%-30 \%$ ratio supplemented by intravenous fentanyl, morphine, Midazolam and Pancuronium. Mechanical ventilation was maintained with a rate of $12-16 \mathrm{~b} /$ $\mathrm{m}$ and tidal volume $8-10 \mathrm{ml} / \mathrm{kg}$. It was readjusted according to blood gas analysis reports. After anesthetic induction and before surgical incision, the central venous line was inserted by the internal jugular venous route, sometimes the subclavian route, inserted usually. Sometimes it was also done under local anesthesia before induction. CVP was recorded and monitored preoperatively.

\section{Monitoring-}

Heart rate, direct arterial blood pressure, central venous pressure, electrocardiography (lead II, aVF, and V5), pulse oxymetry (SPO2), urine output and periodical arterial blood gas analysis were recorded at specific events. (Before and after placement of TEA, before and after induction \& intubation, before and after incision, sternotomy, coronary artery anastomosis, up to skin closure in ICU after extubation and then 60 minutes interval up to $3^{\text {rd }}$ postoperative day. The degree of postoperative analgesia was assessed by clinical well-being, ABG analysis, haemodynamic status and Visual analogue scale (VAS) on day 1, 2 and 3 starting from tracheal extubation.
After completion of skin closure, patients were taken to ICU with epidural catheter (group A) and all monitors attached in ICU. All patients were extubated in the early postoperative period in ICU and the time of extubation and recovery score was noted. Criteria for extubation were a normothermic patient, stable haemodynamic and respiratory conditions and an unremarkable neurological examination. Haemodynamic status was monitored continuously and supports were continued as needed. The time of extubation and recovery score at and $1 \mathrm{hr}$ after extubation was noted.

\section{Operative technique for OPCAB:}

The chest was opened by complete median sternotomy. After careful dissection, LIMA was anastomosed to LAD. After creating a pericardial cradle, the surgeon exposed the target vessels and used traction sutures for mechanical stabilization of the target vessels. Anastomosis was performed in standard beating heart bypass technique using proximal control of the target vessels by stabilizers and a blower mister to clear the anastomotic site.

5000 IU of heparin was administered during LIMA harvesting and half of the total dose of heparin was injected before suturing the aorta. A $75 \%$ dose of Protamine was given at the end of anastomosis. Patients heart were electrically paced if they did not keep a required sinus rhythm $60 \mathrm{~b} / \mathrm{m}$. Dopamine (5-10 $\mu \mathrm{g} / \mathrm{kg} / \mathrm{min})$, adrenaline were used to maintain a mean arterial pressure $>70 \mathrm{~mm} \mathrm{Hg}$ during coronary anastomosis.

\section{Statistical analysis:}

Data were analyzed categorically using the paired student's t test and were expressed as mean + SD. The tests were carried out using the SPSS 11.02 version. A 'p' value $<0.05$ was considered statistically significant.

\section{Results:}

Demographic data and preoperative conditions of the patients are described in table $I$. Demographically there was no significant difference between the two groups in age, height \& weight, co-existing disease and surgical indications. Thirty patients had significant comorbidity. 
Table-I

Demographic characteristics of study population.

\begin{tabular}{lcc}
\hline & $\begin{array}{c}\text { Group A } \\
(\text { TEA+GA) } \\
(\mathrm{n}=20)\end{array}$ & $\begin{array}{c}\text { Group B } \\
(\mathrm{GA} \text { alone) } \\
(\mathrm{n}=20)\end{array}$ \\
\hline Age in yrs & 53.6 & 52.9 \\
Weight in kg & 61.2 & 60.5 \\
Height in cm & 161 & 160 \\
Body surface area $\left(\mathrm{m}^{2}\right)$ & 1.748 & 1.682 \\
LV ejection fraction \% & $35-55$ & $40-55$ \\
Previous MI & 16 & 15 \\
Hypertension (no) & 12 & 14 \\
COPD (no) & 4 & 5 \\
Renal disease (no) & 3 & 2 \\
Peripheral vascular disease (no) & 2 & 0 \\
Diabetes mellitus (no) & 10 & 12 \\
High risk patients & 6 & 4 \\
Surgical indication & & \\
IHD+ SVD (no) & 2 & 4 \\
IHD+DVD (no) & 8 & 12 \\
IHD+TVD (no) & 10 & 4 \\
\hline
\end{tabular}

The records of the haemodynamic parameters of two groups during intraoperative and postoperative periods are shown in fig. and II. HR \& BP were remaining stable throughout the intraoperative and postoperative periods in all pts of group A. In group B pts there were increased intraoperative HR. BP decreased significantly in group $B(p<0.05)$.There was significant difference in the two groups regarding recovery, pain score, requirement of analgesics and patients satisfaction. Time of awakening, movement on command, time of extubation \& appropriate verbal responses were shorter in TEA group than conventional GA group and reached statistically significant level $(\mathrm{p}<0.05)$. Recovery score was higher in group A. VAS scores was lower in group A.

Table-II

Perioperative hemodynamic parameters of study population .

\begin{tabular}{|c|c|c|c|c|c|c|}
\hline & \multicolumn{3}{|c|}{ Group A (TEA+GA) $(\mathrm{n}=10)$} & \multicolumn{3}{|c|}{ Group B (GA alone) $(\mathrm{n}=10)$} \\
\hline & HR beat/min & SBP mm hg & DBP mm of hg & HR beats/min & $\mathrm{SBP} \mathrm{mm}$ of $\mathrm{hg}$ & $\mathrm{DBP} \mathrm{mm}$ of $\mathrm{Hg}$ \\
\hline Preop & $81 \pm 0.78$ & $150 \pm 4.03$ & $88 \pm 3.86$ & $83 \pm 0.99$ & $130 \pm 2.05$ & $80 \pm 5.40$ \\
\hline TEA & $82 \pm 1.41$ & $110 \pm 3.89$ & $60 \pm 1.33$ & $80 \pm 1.24$ & $140 \pm 2.49$ & $75 \pm 1.05$ \\
\hline Intubation & $84 \pm 1.25$ & $105 \pm 6.34$ & $70 \pm 6.43$ & $94 \pm 2.54$ & $150 \pm 3.40$ & $80 \pm 5.40$ \\
\hline Incision & $81 \pm 0.67$ & $120 \pm 4.08$ & $80 \pm 5.40$ & $86 \pm 1.76$ & $180 \pm 2.83$ & $100 \pm 5.60$ \\
\hline Sternotomy & $81 \pm 0.82$ & $110 \pm 5.25$ & $75 \pm 3.02$ & $100 \pm 7.69$ & $170 \pm 4.32$ & $85 \pm 2.62$ \\
\hline Anastomosis & $80 \pm 1.05$ & $100 \pm 1.76$ & $60 \pm 2.54$ & $90 \pm 1.25$ & $130 \pm 3.09$ & $45 \pm 2.91$ \\
\hline Anastomosis & $80 \pm 1.25$ & $100 \pm 2.40$ & $60 \pm 2.54$ & $66 \pm 3.40$ & $70 \pm 3.09$ & $30 \pm 4.08$ \\
\hline After anastomosis & $81 \pm 0.80$ & $120 \pm 4.35$ & $70 \pm 4.99$ & $85 \pm 2.00$ & $130 \pm 3.09$ & $80 \pm 5.40$ \\
\hline Skin closure & $81 \pm 0.78$ & $120 \pm 4.35$ & $80 \pm 5.40$ & $90 \pm 2.54$ & $140 \pm 3.23$ & $90 \pm 2.54$ \\
\hline Arrival in ICU & $80 \pm 0.94$ & $115 \pm 2.94$ & $80 \pm 0.94$ & $94 \pm 1.56$ & $130 \pm 3.09$ & $80 \pm 0.94$ \\
\hline
\end{tabular}

Table-III

Postoperative hemodynamic parameters of study population.

\begin{tabular}{|c|c|c|c|c|c|c|}
\hline & \multicolumn{3}{|c|}{ Group A(TEA+GA) $(n=10)$} & \multicolumn{3}{|c|}{ Group B (GA alone) $(\mathrm{n}=10)$} \\
\hline & HRBeats/min & $\mathrm{SBP} \mathrm{mm}$ of $\mathrm{Hg}$ & DBP $\mathrm{mm}$ of $\mathrm{Hg}$ & HR beats/min & $\mathrm{SBP} \mathrm{mm}$ of $\mathrm{Hg}$ & $\mathrm{DBP} \mathrm{mm}$ of $\mathrm{Hg}$ \\
\hline After extubation & $80 \pm 0.47$ & $110 \pm 3.89$ & $70 \pm 5.77$ & $98 \pm 1.33$ & $140 \pm 2.49$ & $80 \pm 5.40$ \\
\hline 1hr interval & $86 \pm 2.11$ & $100 \pm 1.76$ & $75 \pm 1.05$ & $110 \pm 6.24$ & $120 \pm 4.08$ & $80 \pm 5.40$ \\
\hline $2 \mathrm{hr}$ interval & $82 \pm 1.15$ & $110 \pm 1.76$ & $70 \pm 3.89$ & $94 \pm 2.54$ & $130 \pm 2.05$ & $75 \pm 3.02$ \\
\hline 3hr interval & $81 \pm 0.67$ & $100 \pm 4.08$ & $75 \pm 1.76$ & $105 \pm 0.94$ & $150 \pm 3.40$ & $80 \pm 5.40$ \\
\hline 4hr interval & $84 \pm 1.49$ & $115 \pm 3.89$ & $65 \pm 2.94$ & $92 \pm 3.09$ & $145 \pm 3.43$ & $75 \pm 3.02$ \\
\hline 5 hr interval & $80 \pm 1.05$ & $120 \pm 2.05$ & $70 \pm 4.08$ & $130 \pm 3.77$ & $130 \pm 3.77$ & $70 \pm 4.99$ \\
\hline $6 \mathrm{hr}$ interval & $82 \pm 1.63$ & $110 \pm 1.76$ & $75 \pm 5.25$ & $90 \pm 7.73$ & $160 \pm 3.37$ & $80 \pm 5.40$ \\
\hline 7hr interval & $84 \pm 1.33$ & $110 \pm 5.25$ & $70 \pm 4.99$ & $115 \pm 5.27$ & $140 \pm 3.23$ & $80 \pm 5.40$ \\
\hline 8hr interval & $81 \pm 0.82$ & $120 \pm 4.35$ & $60 \pm 1.33$ & $95 \pm 1.25$ & $120 \pm 4.35$ & $75 \pm 3.02$ \\
\hline 9hr interval & $80 \pm 0.94$ & $100 \pm 2.40$ & $55 \pm 7.39$ & $90 \pm 2.94$ & $130 \pm 3.09$ & $70 \pm 4.99$ \\
\hline
\end{tabular}




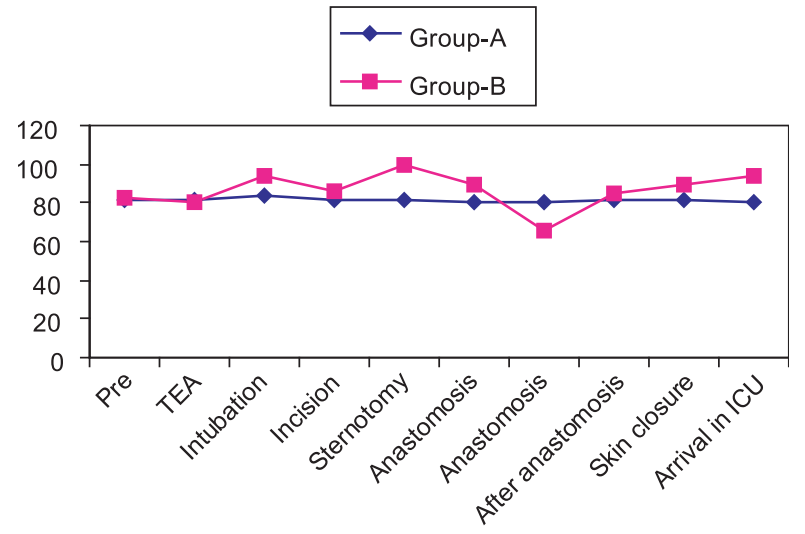

Fig.1: Heart rate from preoperative to arrival in ICU.

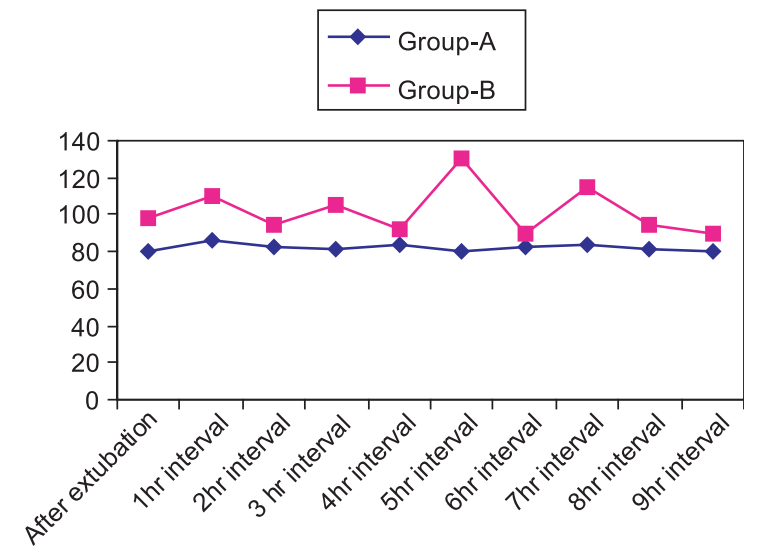

Fig.2: Heart rate from extubation to next 10 hours.

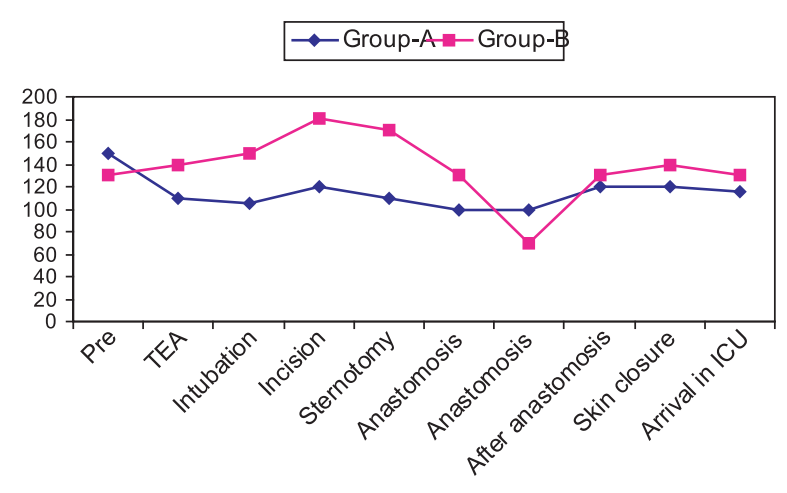

Fig.3: SBP from preoperative to arrival in ICU.

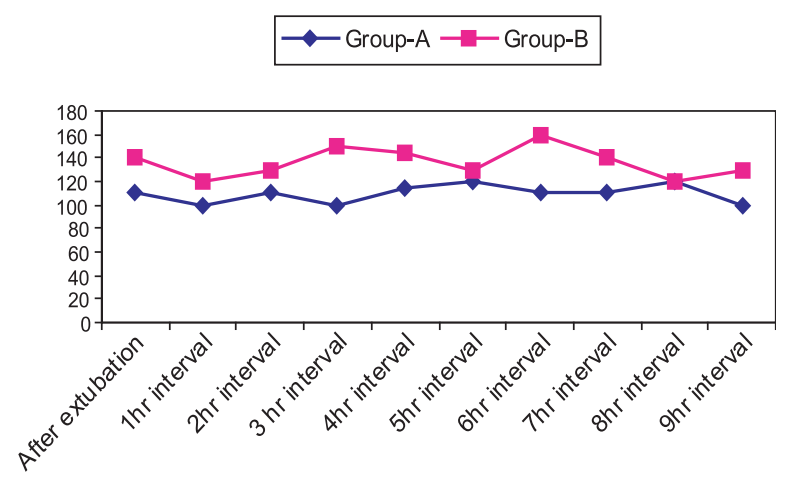

Fig.4: SBP from extubation to next 10 hours.

Table-IV

Recovery Score of study population.

\begin{tabular}{lcccc}
\hline & Consciousness & $\begin{array}{c}\text { Activity } \\
\text { (Limb movement) }\end{array}$ & $\begin{array}{c}\text { Time to respond } \\
\text { of vocal command }\end{array}$ & $\begin{array}{c}\text { Time to } \\
\text { extubation }\end{array}$ \\
\hline Group A & 2 & 2 & $1.81 \pm 0.26$ & $3.04 \pm 0.66$ \\
Group B & 1 & 1 & $4.05 \pm 0.31$ & $7.62 \pm 0.78$ \\
\hline
\end{tabular}

Table-V

Analgesia Score of study population.

\begin{tabular}{lccccc}
\hline & & VAS & & $\begin{array}{c}\text { Analgesic requirement } \\
\text { within 3 days }\end{array}$ & Pt's satisfaction \\
& $\mathrm{I}^{\text {st }}$ POD & $2^{\text {nd }}$ POD & $3^{\text {rd }}$ POD & & \\
\hline Group A & $2 \pm 1.19$ & $1.56 \pm 0.95$ & $1.28 \pm 0.71$ & 0 & $80 \%$ \\
Group B & $6.0 \pm 0.4$ & $4.26 \pm 0.80$ & $3.44 \pm 0.20$ & $4.4 \pm 0.96$ & $40 \%$ \\
\hline
\end{tabular}

All these parameters showed significant difference between group A and group B by student's t test (table II \& III).

There is also lower incidence of arrhythmia, hypotension, in group A so less requirement of inotrops, vasopressor, and IV fluid. Perioperative bleeding was also less in TEA group, so negligible requirement of blood transfusion. In TEA group, better peri and postoperative analgesia improves pulmonary functions, less incidence of chest 
infection, postoperative hypoxemia, shorter period of ventilation so early mobilization and short length of ICU stay. There is also lower incidence of nausea vomiting among group A patients receiving TEA plus standard GA.

\section{Discussion:}

We have demonstrated from this study that TEA plus General anesthesia in patients with CAD for OPCAB allows faster and better recovery and better postoperative analgesia comparable to standard GA alone.

Procedures to minimize surgical, anaesthesiologic or cardiopulmonary bypass trauma during cardiac operations are in continuous development in an effort to reduce postoperative morbidity and mortality. The current technique of beating heart coronary artery bypass grafting (CABG) is intended to decrease the adverse side effects typically associated with cardiopulmonary bypass ${ }^{10}$ resulting in reduced morbidity and reduced length of hospital stay. Off-pump coronary revascularization is an old technique performed first in St. Petersburg in 1964, 62 but was soon outshone by the rapid development of CPB and cardioplegia. ${ }^{3}$ A revival of the technique occurred during 1980s. ${ }^{3}$ Recent improvement in the technique has resulted in the possibility of multiplevessel grafting in all coronary territories with a graft patency comparable with conventional surgery.

During beating heart surgery, the maintenance of haemodynamic stability during heart enucleation necessary for accessing each coronary artery and optimum myocardium protection during ischemic events is the primary goal of anesthetic management.

Four decades of clinical research have failed to find a general anesthetic technique that attenuates the stress response and the resultant adverse sequelae associated with surgery and $\mathrm{CPB}^{11}$. Spinal cord anesthesia prevents the surge in stress hormones that accompanies cardiac procedures. TEA provides excellent conditions for OPCAB surgery by dilating the coronary arteries and the LIMA and by reducing heart rate and arrhythmia during manipulation of the heart. ${ }^{12-14}$ And also results in reduced coronary vascular resistance, reduced perioperative myocardial ischemia, reduced internal thoracic artery spasm, reduced systemic vascular resistance, reduced platelet activation and inflammation. ${ }^{15}$

Epidural anesthesia had proved to be very efficient in achieving somatosensory and motor block in the chest, which in turn enabled the performance of CABG in very light plane of general anesthesia, particularly in high risk patients such as very elderly, EF > $30 \%$, chronic obstructive pulmonary disease, coagulating disorders and aberrant neurological condition. Besides these intraoperative advantages, postoperative pain management is facilitated by continuous epidural application of analgesic agents. Such effective pain management improves postoperative pulmonary function, decreases postoperative Ileus resulting in early mobilization and recovery. Rapid postoperative mobilization and faster recovery shortens hospitalization time and total cost and may open the door for outpatient CABG surgery even for multivessel coronary artery disease. TEA in OPCAB achieved excellent patient acceptance by avoiding cardiopulmonary bypass and allowing effective pain management.

The most dreaded complication of thoracic epidural anesthesia is the epidural hematoma formation, which is estimated to be very low. In 2000, Ho et al. used mathematical modeling to calculate the statistical risk of epidural hematoma following TEA as between 1 in $150,000^{16-17}$ and 1;1,500 (95\% confidence) and up to $1 ; 1,000$ (99\% confidence) based on published data available at that time. They also acknowledged the importance of factors including presence of a normal coagulation system prior to insertion and removal of the catheter, avoidance of repeated attempts, postponing surgery in the presence of a bloody tap and closed neurological surveillance in minimizing risk. ${ }^{18}$ These precautions are repeated in most studies. Also, eight studies inserted the epidural catheter the day before surgery. We also found no such complication during this study.

From the above discussion, we conclude that the use of TEA in general anesthesia results in more rapid extubation and significant better pain relief in patients undergoing OPCAB surgery. There may also be a lower incidence of respiratory, cardiovascular and renal complication. 


\section{Conclusion:}

It can be concluded that the use of TEA in GA results in more rapid extubation and significantly better pain relief in patients undergoing CABG surgery. There may also be a lower incidence of respiratory, cardiovascular and renal complication.

\section{Conflict of Interest - None.}

\section{References:}

1. Mark MJ, Pfizer A, Bachand D et al. Comparison of coronary artery bypass surgery with and without Cardiopulmonary bypass in patient with multivessel disease. J Thorac Cardiovasc Surg 2004; 127:167-73.

2. Demaria RG, Carrier M, Fortier S, et al. Reduced mortality and stroke with off pump coronary artery bypass grafting surgery in octogenarians. Circulation 2002; 106: 15-0.

3. Gary Okum and Jay C. Horrow. Anesthetic management of myocardial revascularization: $A$ practical Approach to Cardiac Anesthesia: Anaesthesia management of myocardial revascularizatio $\left(4^{\text {th }}\right.$ edition): 2008: 289-315.

4. P.G Chassot, P. van der Linden, M. Zaugg et al. Offpump coronary artery bypass surgery: physiology and anaesthetic management. British Journal of Anaesthesia 2004;92(3): 400-413.

5. Diegeler A, Matin M, Falk V, Battellini R, Walther T, Autschbach R, et al. Coronary bypass grafting without cardiopulmonary bypass- techniqual considerations, clinical results, and follow up. Thorac Cardiovasc Surg 1999; 47:14-8.

6. Calafiore AM, Vitolla G, Iovino T, Iaco Al, Mazzei V, Commodo M. Left anterior small thoracotomy (LAST): mid-term results in single vessel disease. J Card Surg 1998; 13:306-9.

7. Diegeler A, Matin M, Falk V, Battellini R, Walther T, Autschbach R, et al. Coronary bypass grafting without cardiopulmonary bypass- techniqual considerations, clinical results, and follow up. Thorac Cardiovasc Surg 1999; 47:14-8.
8. Riedel BJ, Wright IG. Epidural anesthesia in coronary artery bypass grafting surgery. Curr Opin Cardiol 1997;12:515-21.

9. DavC. H. Cheng. Regional analgesia and ultra-fast-track cardiac anaesthesia. Canadian Journal of Anaesthesia 2005; 52: 12-17.

10. Fillinger MP, Yeager MP, Dodds TM, Fillinger MF, Whalen PK, Glass DD. Epidural anaesthesia and analgesia: Effect on recovery from cardiac surgery. $J$ Cardiothorac Vasc Anesth 2002; 16:15-20.

11. Stenseth R, Bjella L, Berg EM, et al. Effects of thoracic epidural analgesia on pulmonary function after coronary artery bypass surgery. Eur J Cardiothorac Surg 1991; 10:859-65.

12. Steneth R. Thoracic epidural analgesia in aortocoronary bypass surgery; hemodynamic effects and endocrine metabolism. Acta Anesthesiol Scand 1994; 38:826-39.

13. Tenling A, Joachimsson PO, Tyden H, Hedentrierna G. Thoracic epidural analgesia as an adjunct to general anaesthesia for cardiac surgery. Acta Anesthesiol Scand 2000; 44:1071-6.

14. Blomberg S, Emanuelsson H, Kvist H, Lamm C, Ponten J, waagstein F et al. Effects of thoracic epidural anesthesia on coronary arteries and arterioles in patients with coronary artery disease. Anaesthesiology 1999; 73:840-7.

15. Fawcett WJ, Edwards RE, Quinn AC, MacDonald IA, Hall GM. Thoracic epidural analgesia started after cardiopulmonary bypass. Adrenergic, cardiovascular and respiratory sequelae. Anaesthesia 1997; 52:914-6.

16. Ho AM, Chung DC, Joynt GM. Neuroaxial blockade and hematoma in cardiac surgery: estimating the risk of a rare adverse that has not (yet) occurred. Chest $2000 ; 117: 551-5$

17. Renck H. Neurological complications of central nerve blocks. Acta Anesthesiol Scand 1995; 39:859-68.

18. Ronald A, AbdulAziz A. K, Day TG, Scott M. In patients undergoing cardiac surgery, thoracic epidural analgesia combined with general anaesthesia results in faster recovery and fewer complications but does not affect length of hospital stay. Interact Cardiovasc Thorac Surg 2006; 5:207-216. 